\title{
Need satisfaction, motivational regulations and exercise: moderation and mediation effects
}

\author{
Karin Weman-Josefsson ${ }^{1,2,4^{*}}$, Magnus Lindwall ${ }^{2,3,4}$ and Andreas Ivarsson ${ }^{1}$
}

\begin{abstract}
Background: Based on the Self-determination theory process model, this study aimed to explore relationships between the latent constructs of psychological need satisfaction, autonomous motivation and exercise behaviour; the mediational role of autonomous motivation in the association of psychological need satisfaction with exercise behaviour; as well as gender and age differences in the aforementioned associations.

Methods: Adult active members of an Internet-based exercise program $(n=1091)$ between 18 and 78 years of age completed a test battery on motivational aspects based on Self-determination theory. The Basic Psychological Needs in Exercise Scale and the Behavioural Regulation in Exercise Questionnaire-2 were used to measure need satisfaction and type of motivation and the Leisure Time Exercise Questionnaire to measure self-reported exercise.

Results: Need satisfaction predicted autonomous motivation, which in turn predicted exercise, especially for women. Autonomous motivation was found to mediate the association between need satisfaction and exercise. Age and gender moderated several of the paths in the model linking need satisfaction with motivation and exercise.

Conclusion: The results demonstrated gender and age differences in the proposed sequential mechanisms between autonomous motivation and exercise in the process model. This study thus highlights a potential value in considering moderating factors and the need to further examine the underlying mechanisms between needs, autonomous motivation, and exercise behaviour.
\end{abstract}

Keywords: Mediation, Moderation, Motivation, Self-determination

The positive effects of physical activity (PA) on health are well established, and it is generally accepted that regular PA and exercise can be used to prevent and treat a variety of physical and psychological diseases [see The European Health Reports: $[1,2]$ and to have beneficial effects on physical and psychological wellbeing [3]. A decade ago, Bull, Armstrong [4] stated that the effective promotion of a more physically active lifestyle has the potential to prevent as many as two million premature deaths and nearly 20 million disability-adjusted life years (DALYs) worldwide. Still, almost ten years later, health statistics show discouragingly low levels of PA and exercise levels (see [5]), and many interventions promoting PA and exercise are considered ineffective $[6,7]$. Mounting

\footnotetext{
* Correspondence: karin.weman@hh.se

'Research on Welfare, Health and Sport, Halmstad University, PO Box 823, SE 30118 Halmstad, Sweden

2Department of Psychology, University of Gothenburg, PO Box 500, SE 405

30 Gothenburg, Sweden

Full list of author information is available at the end of the article
}

literature strongly advocates the use of sound theory application in order to improve intervention efficacy (e.g., $[8,9])$, and a lack of studies explaining the underlying processes (i.e., mechanisms) of theoretically derived hypotheses may partially account for many intervention failures [7]. In order to design successful interventions, an understanding of mediation models is fundamental for comprehending the complex interactions between theoretical constructs (e.g., motivation) and behaviour [7]. A widely used theory in modern research on human motivation is selfdetermination theory (SDT; $[10,11])$, a framework that has received substantial support for its usefulness in health behaviour change (e.g., [12]) and in understanding exercise and PA behaviour (e.g., $[13,14])$, as well as regarding proposed mechanisms of health behaviour change $[15,16]$.

Self-determination theory is a multidimensional theory based on the importance of people being self-determined in order to be motivated and engaged in certain behaviours 
[11]. A fundamental notion in SDT is that different types of motivation differ qualitatively along a continuum, in relation to the degree of self-determination or the extent to which the behaviour is regulated by controlling aspects. These relationships are described in a sub-theory called organismic integration theory (OIT; [17]). Amotivation represents one end of the continuum and is a lack of any intention to engage in the behaviour. At the other end of the continuum lies intrinsic motivation, the most self-determined, or autonomous, form of motivation. When intrinsically motivated, a person performs the behaviour volitionally because it feels inherently interesting or enjoyable. Extrinsic motivation, situated between amotivation and intrinsic motivation on the continuum, instead regulates the behaviour in order to achieve outcomes separate from the behaviour itself. There are four types of extrinsic motivation (external, introjected, identified and integrated regulation), which denote progressively more self-determined motives in relation to the degree to which the behaviour has been internalized. Internalization is a process where people integrate (social) values and behaviours into the self [17] and in this manner extrinsic motivation can become more self-determined (autonomous) when the behaviour is performed in order to achieve internalized outcomes [13]. As an example, integrated regulation signify a high level of internalization (social regulations fully integrated to the self), while introjection signify only partially internalized regulations [17]. With increased internalization, the motivation becomes more self-determined, which enhances persistence and adherence and internalization is of particular importance in maintaining nonenjoyable behaviours like exercise [11].

Another sub-theory of SDT, the basic needs theory (BNT), holds that self-determined motivation originates from individuals' innate tendency to satisfy three basic psychological needs: competence, relatedness, and autonomy [17]. The need for competence denotes the feeling of effectance and capability, while autonomy represents feelings of volition or self-determination and relatedness refers to feelings of social inclusion and closeness. Ryan and Deci [17] define these basic psychological needs as "innate psychological nutriments that are essential for on-going psychological growth, integrity and well-being" (p 229) and suggest they are essential for understanding the content (what) and process (why) of human goals and behaviours. According to SDT $[17,18]$ and extensions of the SDT model $[18,19]$, self-determined motivation will be promoted when the three needs are satisfied. Also, it is proposed that self-determined motivation will lead to important behavioural, affective and cognitive outcomes, while the consequences are decreasingly positive from introjected and external motivation to amotivation [11]. A common trend in previous work is the strong inter-correlations between the three needs; and between competence and autonomy in particular (e.g., [20]), suggesting that the three needs may be captured by an underlying unidimensional factor. Supporting this view, Hagger, Chatzisarantis [21] found that a single global need satisfaction factor could explain latent variables representing autonomy, competence and relatedness.

A proposed key assumption [22] is that self-determined motivation mediates the association between need satisfaction and behavioural outcomes, also illustrated by the SDT process model proposed by [23]. Specifically linked to PA and exercise contexts, the model posits that a higher degree of satisfaction of needs (related to the behaviour) is suggested to be associated with increased exercise through a more self-determined motivation [22]. This relationship is supported by a considerable amount of research [13-15], also longitudinally in experimental designs [24], but the literature on how this link between needs, self-determined motivation and behavioural outcomes such as PA and exercise actually operates is somewhat inconsistent. Some studies [25] have found that need satisfaction and/or self-determined motivation are related to PA/exercise behaviour, whereas others (e.g., [26]) have found that self-determined motivation is unrelated to these behaviours. One reason for these different results could be the influence of various moderating factors (e.g., gender and age) on the associations between needs, motivation and behaviour. In addition, although several studies have supported the different individual paths of this proposed mediating model, few have fully tested the key assumption that self-determined motivation will mediate the association between need satisfaction and outcomes in the context of PA and exercise using recommended analyses also considering moderating effects. Edmunds and Ntoumanis [25], for example, found that the relationship between the need for competence and strenuous exercise was partially mediated by selfdetermined motivation (identified regulation). A limitation in their study, though, is that they used the simpler analytical regression strategy of Baron and Kenny [27], a method not recommended in modern research (see $[28,29]$, for example due to its conservative nature and low power [8]. McDonough and Crocker [26] used structural equation modelling (SEM), testing the mediation hypothesis, and found that the satisfaction of all three needs was related to self-determined exercise motivation but that self-determined motivation only partially mediated the effect on positive and negative affect and was unrelated to the behavioural outcome. The SEM analyses enable examination of measurement-free associations between constructs and more robust mediational paths.

In addition, neither of these two previous studies examined possible moderating factors such as gender or age, or the relationships between needs, motivation and outcomes, which may explain when and for whom need 
satisfaction and self-determined motivation may be related to outcomes and when and for whom self-determined motivation may mediate the proposed effect of need satisfaction on outcomes. This line of research questions has been raised as an important issue for future research $[28,29]$. In a recent review on SDT in exercise and PA [14], more sophisticated analyses were specifically requested in order to clarify the role of need satisfaction in the development of self-determined motivation and to study possible moderating factors like gender and age differences. Following these recommendations, and considering that both age and gender could reasonably play an important role in the motivational processes within the SDT context, these moderating factors should be taken into account when studying these processes. Although basic psychological needs are thought to be universal and apply across genders, ages and cultures, it is likely that such factors could influence the means by which basic needs are met [17], how well the social context support need fulfilment, as well as how behavioural regulations emerge. Over the course of a lifetime, people's reasons for engaging in exercise and PA may change along with aspects like natural variations in things like values, health and goals [30]. For example, older adults tend to have more intrinsically oriented exercise goals and motives [31], suggesting that they may have a more autonomous exercise motivation. Research findings regarding gender differences are mixed. Some studies imply that women have a general tendency towards more controlled regulations (mainly introjected) than men [14] while some suggest the opposite, that women are more autonomously regulated and men more externally regulated to exercise behaviour [32]. At the same time, a meta-analysis found only negligible gender differences in motivational regulations [33]. These mean-level results suggest that there is reason to also expect possible moderators in the pathways between need satisfaction, regulation and outcomes (such as gender and age differences). The identification of such moderators would provide important information for the theoretical understanding of SDT-based models of exercise [14].

The main aim of this study was to explore: (a) theoretically derived hypotheses about the relationships between the latent (free of measurement error) constructs of psychological need satisfaction, autonomous motivation, and the manifest variable of exercise behaviour; (b) the mediational role of autonomous motivation in the association of psychological needs with exercise behaviour; and (c) gender and age differences (moderating effects of gender and age groups) in the aforementioned associations.

\section{Methods}

Participants

The participants $(N=1091)-286$ men and 805 women, aged 18-78 years $(M=45.0 ; S D=11.7)$-were all active Swedish members of an Internet-based exercise programme created by a Swedish company in the e-health industry offering web-based health-care services (e.g., pedometer step contests, weight-loss programmes, etc.) mainly in the private sector. Hence, the sample was expected to be diverse regarding, for instance, fitness level, age and gender, as well as motivational aspects.

\section{Measures}

The Basic Psychological Needs in Exercise Scale (BPNES; [34]) measures satisfaction of the three needs autonomy, competence and relatedness in the exercise domain through 12 items (e.g., "The way I exercise is in agreement with my choices and interests") and a five-point Likert scale, where 1 = "I don't agree at all" and $5=$ "I completely agree". Cronbach's alpha for the BPNES was 0.81 to 0.92 in the present study. The BPNES has been successfully validated as supporting the theoretically based threefactor model and the needs hypothesis of SDT [34]. It has also demonstrated gender invariance [35]. Motivation quality was measured by the Behavioural Regulation in Exercise Questionnaire-2 (BREQ-2; [36]) which has 19 items (e.g., "It's important to me to exercise regularly") and a five-point Likert scale, where $0=$ "not true for $m e$ " and $4=$ "very true for $m e$ ". The present study applied a four-pointed Likert with the same anchors, i.e., 1 = "not true for $m e$ " and $4=$ "very true for $m e$ " scale. The scale measures behavioural regulations through five factors: extrinsic, introjected, identified and intrinsic motivation, and, unlike the original BREQ, the BREQ-2 also measures amotivation. In addition to using the five different regulations in BREQ-2 we followed the example of Sebire, Standage [37], and created one controlled motivation factor (external regulation and introjected regulation) and one autonomous motivation (identified regulation and intrinsic motivation) factor. Cronbach's alpha for the BREQ-2 factors were 0.73 to 0.86 in the present study. The Leisure Time Exercise Questionnaire [38] was used to measure self-reported exercise. The LTEQ consists of three questions regarding the frequency of performing strenuous, moderate and light exercise during a regular week. The total exercise score can be calculated and transformed into the metabolic equivalent of exercise (MET) scores.

The BPNES and BREQ-2 were translated from English into Swedish according to the back-translation method [39]. A bilingual (English and Swedish) expert first translated the tests from English into Swedish, and then another bilingual expert translated them back into English. Differences in the translated versions and the originals were discussed in the research group and formed the foundation of the final versions. For the invariance and moderation analyses, mean age (45.0) was used to create 
two age groups: a younger (18-45 years) and an older (46-78 years) one.

When testing the factor validity and invariance of the translated BPNES and BREQ-2 scales, the theoretical a priori models displayed adequate-to-good fit with data. For the BPNES, the theoretical a priori three-factor model demonstrated good fit with data: Satorra-Bentler $\chi 2=246.45$ (51df), $C F I=0.96 ; R M S E A=0.059$ (0.052-0.067). The fivefactor model of the BREQ-2 demonstrated acceptable fit to data: $\chi^{2}=408.60$ (142df), $C F I=0.94 ; R M S E A=0.044$ (0.039-0.049). All standardized factor loadings were significant and generally over .60 . The three-factor measurement model of the BPNES displayed strict invariance (i.e., invariant residuals in addition to invariant factor loadings and intercepts) across gender and age, as the CFI did not decrease more than .01 in model fit when factor loadings, intercepts, and residuals were constrained to be equal across groups of gender (men and women) and different ages (18-45 years, and 46-78 years). The BREQ- 2 measurement model demonstrated strong invariance (i.e., invariant factor loadings and intercepts) across gender and across age groups.

\section{Procedures}

The study began with a pilot study including ten persons selected through convenience sampling to test the comprehension and design of the test battery, using the think-aloud method [40]. The pilot study resulted in the clarification and remodelling of parts of the test battery for the final version. Following a list of members provided by the e-health service company, potential participants for Study 1 were contacted by e-mail, with information on the aim of the study, ethical concerns and practical issues. When logging in to the questionnaire, the participants had to tick a box for informed consent in order to access the questionnaire The collected data were stored in a certain web account accessible only by the researchers. Participation was anonymous, and no personal data were requested; hence, no personal register was created. The study was approved by the regional ethical board.

\section{Analysis}

$T$-tests were conducted to examine differences in psychological need and motivation across gender and agegroups., In the main analyses, structural equation modelling (SEM) and mediation analysis using a bootstrapping resampling approach $[28,41]$ were used, enabling the examination of measurement-free associations between constructs and more robust mediational paths. Mplus version 7.1 (Muthen \& Muthen, 1998-2009) was used to analyse the data with the robust maximum likelihood (MLR) estimators. Missing data were handled using a full maximum likelihood (FIML) estimator, which is default in Mplus. Therefore, data from all $(N=1091)$ participants (i.e., including those who had missing data on some items or variables) were used in the Mplus analyses. Based on recommendations by $\mathrm{Hu}$ and Bentler [42], the following fit indexes were used: (a) SatorraBentler chi-square statistics, (b) Bentler's comparative fit index (CFI; [43]), and (c) the root mean square error of approximation (RMSEA; [44]). For the CFI, values close to or greater than 0.95 indicate a well-fitting model [42]. For the RMSEA, values less than .05 indicate a good fit, whereas values up to .08 represent a reasonable fit [44]. In the invariance testing, we used the recommendations by Cheung and Rensvold [45]. Because the chi-square difference test is sensitive to the sample size, they recommend using a decline in the CFI of 0.01 or less as indicative of invariance. Moderation analyses were conducted through multi-group analyses, whereby model fit for models with no constraints between groups (e.g., men vs. women) in terms of paths was compared with model fit in models in which certain paths were constrained to be equal between groups.

Mediator models with a bootstrapping resampling approach for calculating product-of-coefficients and asymmetric $95 \%$ confidence intervals based on 1000 resamples $[29,41]$ were used to test indirect effects. All mediation analyses were performed in Mplus, using latent variables for the need satisfaction (BPNES) and motivation (BREQ-2) variables. Moreover, bias-corrected and accelerated bootstrap confidence intervals for the indirect effects were used [29]. Bootstrap confidence intervals are recommended because they do not make unrealistic assumptions about the shape of the sampling distribution of the indirect effect like, for example, the Sobel test does [29]. In the analyses, indirect effects of the independent variable (in the present study, psychological need satisfaction) on the outcome variable (exercise) through the proposed mediator variables (e.g., autonomous motivation) were estimated (see Fig. 1). The Wald Chi-Square test in Mplus was used to test if gender or age moderated the mediational effects of motivation.

\section{Results}

Descriptive data divided into gender and age groups are presented in Table 1 . The $t$-tests revealed men to report higher external regulation $(t(1054)=2.04, p<.05)$, higher introjected regulation $(t(1046)=2.11, p<.05)$ and more strenuous exercise $(t(1010)=2.52, p<.05)$, but less moderate exercise $(t(1012)=-3.95, p<.05)$ compared to women. Turning to age differences, younger adults reported higher external $(t(1018)=2.35, p<.05)$ and introjected $(t$ $(1000)=5.93, p<.01)$ regulation, and were also engaged in more strenuous $(t(968)=4.9, p<.01)$ and total exercise $(t(966)=2.56, p<.01)$ than older adults, who instead were more engaged in moderate exercise $(t(966)=2.56$, $p<.01)$ than younger adults. 


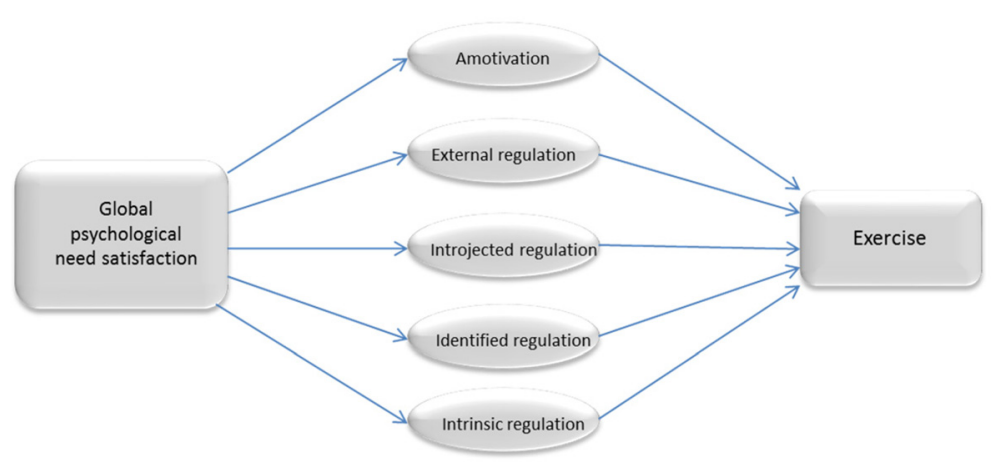

Fig. 1 The structural equation model used to examine the relations between psychological need satisfaction, motivational regulations, and exercise

\section{Psychological need satisfaction and controlled and autonomous motivation predicting exercise}

When using the three need satisfaction factors simultaneously to predict motivation in the analyses, we found that whereas competence and relatedness predicted autonomous motivation in expected positive directions $(\beta=.89$, $p<.01$ and $\beta=.15, p<.01$ respectively) the path between autonomous need satisfaction and autonomous motivation was negative and significant $(\beta=-.33, p<.01)$, which was unexpected. Given that the correlations between autonomy and the latent factors of the BREQ-2 were according to expectations (i.e., positive correlations with identified and intrinsic regulation but negative correlations with amotivation and external regulation), the negative path displayed in the model between autonomy and autonomous motivation most probably signals a suppressor effect rather than a conceptually meaningful result. Because the latent factors of competence, autonomy and relatedness correlated moderately to strongly, the three psychological need satisfaction factors were collapsed into one total psychological need factor, using a second-order (higher-order) model.

In the first step, a model including the total psychological need satisfaction factor predicting autonomous and controlled motivation, which in turn predicted exercise behaviour (METS), was tested. The autonomous

Table 1 Descriptives (means and standard deviations) of psychological need satisfaction (BPNES), behavioural regulations (BREQ-2), and exercise (LTEQ) last six months (PA), across gender and age groups

\begin{tabular}{|c|c|c|c|c|c|}
\hline & \multicolumn{2}{|l|}{ Gender } & \multicolumn{3}{|l|}{ Age } \\
\hline & Men & Women & Younger & Older & Total \\
\hline & $(n=286)$ & $(n=805)$ & $(18-45)$ & $(46-78)$ & sample \\
\hline & & & $(n=539)$ & $(n=501)$ & $(N=1091)$ \\
\hline \multicolumn{6}{|l|}{ BPNES } \\
\hline Autonomy & $4.0(.84)$ & $4.1(.80)$ & $4.0(.81)$ & $4.1(.81)$ & $4.1(.81)$ \\
\hline Competence & $3.8(.84)$ & $3.8(.82)$ & $3.8(.82)$ & $3.8(.83)$ & $3.7(.82)$ \\
\hline Relatedness & $3.9(1.1)$ & $3.8(1.0)$ & $3.8(1.00)$ & $3.8(1.09)$ & $3.8(1.0)$ \\
\hline Global need & $11.7(2.3)$ & $11.7(2.3)$ & $11.6(2.2)$ & $11.7(2.3)$ & $11.7(2.3)$ \\
\hline \multicolumn{6}{|l|}{ BREQ-2 } \\
\hline Amotivation & $1.1(.30)$ & $1.1(.24)$ & $1.1(.28)$ & $1.1(.23)$ & $1.1(.25)$ \\
\hline External reg. & $1.2(.39)$ & $1.1(.34)$ & $1.2(.38)$ & $1.1(.32)$ & $1.1(.35)$ \\
\hline Introjected reg. & $2.1(.73)$ & $2.2(.78)$ & $2.3(.75)$ & $2.0(.77)$ & $2.2(.77)$ \\
\hline Identified reg. & $3.2(.66)$ & $3.2(.63)$ & $3.2(.65)$ & $3.2(.62)$ & $3.2(.63)$ \\
\hline Intrinsic reg. & $3.2(.70)$ & $3.3(.69)$ & $3.3(.70)$ & $3.3(.67)$ & $3.3(.70)$ \\
\hline \multicolumn{6}{|l|}{ LTEQ } \\
\hline Strenuous & $2.2(1.8)$ & $1.8(1.6)$ & $2.1(1.9)$ & $1.6(1.5)$ & $1.9(1.7)$ \\
\hline Moderate & $2.9(3.5)$ & $3.7(2.6)$ & $3.3(2.4)$ & $3.7(3.3)$ & $3.5(2.9)$ \\
\hline Light & $3.6(4.1)$ & $3.8(2.9)$ & $3.8(3.2)$ & $3.6(3.3)$ & 3.7 (3.3) \\
\hline
\end{tabular}

global need = total need satisfaction factor; reg = regulation 
factor consisted of the BREQ-2 items hypothesized to load on the identified regulation and intrinsic motivation factors whereas the controlled motivation factor were comprised of the items hypothesized to load on the external regulation and introjected regulation factors. Collapsing items this way to create the autonomous and controlled motivation factors resulted in a suboptimal fit according to the CFI, but reasonable fit according to the RMSEA for the full group: Satorra-Bentler $X 2$ (345) = 2019.90, $p<.001 ; C F I=.86$; RMSEA $=.067$ (90 \% CI $=.064$ to .070$)$. The results from the model are presented in Table 2. The path from total psychological need satisfaction to controlled motivation was weakly negative and significant $(\beta=-.26, p<.01)$, whereas the path from need satisfaction to autonomous motivation was strongly positive and significant $(\beta=.68, p<.01)$.

In the next step, the two factors controlled and autonomous motivation were replaced with the five factors of the BREQ-2, to offer more specific insight into how various types of motivation regulation were associated with total need satisfaction and exercise behaviour. This model is illustrated in Fig. 1. For the full sample (see Table 3, last column to the right), total need satisfaction, as modelled by the higher-order factor, significantly and inversely predicted amotivation $(\beta=-.44, p<.01)$ and external regulation $(\beta=-.26, p>.01)$ but not introjected regulation. Moreover, total need satisfaction predicted both identified regulation $(\beta=.79, p<.01)$ and intrinsic motivation $(\beta=.81, p<.01)$. In terms of the paths from type of motivation to exercise, only identified regulation significantly $(\beta=.30, p<.01)$ predicted exercise behaviour for the full sample.

We also examined the relationships of different exercise intensities with psychological need satisfaction and motivation regulation, modelled as latent constructs. The results are described in Table 4. In general, the correlations between self-reported light exercise intensity with needs and regulations were very small and non-

Table 2 Predictions between psychological need satisfaction, motivational type (controlled vs. autonomous motivation) and exercise across gender and age groups (standardized estimates)

\begin{tabular}{llllll}
\hline Regression weights & Men & Women & $\begin{array}{l}\text { Younger } \\
(18-45)\end{array}$ & $\begin{array}{l}\text { Older } \\
(46-78)\end{array}$ & Full sample \\
& $n=286$ & $n=805$ & $n=539$ & $n=550$ & $N=1091$ \\
\hline Need $\rightarrow$ Contmot & $-.20^{*}$ & $-.29^{* *}$ & $-.21^{* *}$ & $-.30^{* *}$ & $-.26^{* *}$ \\
Need $\rightarrow$ Autmot & $.72^{* *}$ & $.66^{* *}$ & $.66^{* *}$ & $.70^{* *}$ & $.68^{* *}$ \\
Contmot $\rightarrow$ Exercise & $.26^{* *}$ & -.07 & -.03 & .08 & .02 \\
Autmot $\rightarrow$ Exercise & $.23^{*}$ & $.35^{* *}$ & $.35^{* *}$ & $.31^{* *}$ & $.33^{* *}$ \\
\hline
\end{tabular}

Need $=$ total need satisfaction factor; Contmot $=$ Controlled motivation (combined external and introjected regulation); Autmot = Autonomous motivation (combined identified regulation and intrinsic motivation) ${ }^{*} p<.05$; ${ }^{* *} p<.01$. A complete table with standard errors for the estimates can be obtained by request from the first author significant. Moderate exercise intensity was weakly but significantly associated with the needs autonomy $(\Phi=.17$, $p<.01)$ and competence $(\Phi=.19, p<.01)$ and with amotivation $(\Phi=-.11, \mathrm{p}<.01)$, identified regulation $(\Phi=.11$, $p<.01)$ and intrinsic motivation $(\Phi=.14, p<.01)$. Global need was weakly correlated with moderate exercise $(\Phi=-.18, \quad \mathrm{p}<.05)$, but moderately correlated with strenuous $(\Phi=.43, p<.01)$ and total $(\Phi=.35, p<.01)$ exercise. Strenuous exercise intensity demonstrated the most robust pattern of relationships with needs and regulations with significant associations with all three needs and all five regulations. The correlations with the three needs and identified regulation and intrinsic motivation were moderate in size $(\Phi=.47-.31, p<.01)$, whereas the correlation with amotivation $(\Phi=-.17, \mathrm{p}<.01)$ and extrinsic regulation $(\Phi=-.11, \mathrm{p}<.01)$ were weak and negative. Mirroring how the total exercise score of METS is calculated (putting heavy focus primarily on frequency of strenuous exercise), the correlations of total exercise score with needs and regulations were slightly lower than for strenuous exercise, but higher than for moderate and light exercise.

\section{Moderating effects of age and gender}

The moderating effects analyses were conducted using the total exercise score. Only one significant difference across age groups or gender was found in the model including controlled and autonomous (see Table 3). Controlled motivation positively predicted exercise for men $(\beta=.26$, $p<.01)$ but not for women $(\beta=-.07, p=.07)$, mirrored by a significant decrement in model fit $\left(\Delta \chi^{2}=13.78 / 1 d f\right)$ when these paths were constrained to be equal across men and women.

When including the five separate BREQ-2 factors in the model, a number of significant differences in strength and direction of paths between men and women appeared (see Table 4). Model fit decreased significantly (reflecting a significant difference between men and women) when the following paths were constrained to be equal: total need satisfaction to introjected regulation $\left(\Delta \chi^{2}=24.06 / 1 d f\right)$; total need satisfaction to identified regulation $\left(\Delta \chi^{2}=4.09\right.$ / $1 d f)$; external regulation to exercise $\left(\Delta \chi^{2}=9.84 / 1 \mathrm{df}\right)$; introjected regulation to exercise $\left(\Delta \chi^{2}=11.67 / 1 d f\right)$; and identified regulation to exercise $\left(\Delta \chi^{2}=28.02 / 1 d f\right)$. Looking more specifically into these differences, the path between total need satisfaction and introjected regulation was positive and significant for men $(\beta=.41, p<.01)$ but negative and non-significant for women, and total need satisfaction was more strongly related to identified regulation for men $(\beta=.88, p<.01)$ than for women $(\beta=.75$, $p<.01)$. Moreover, external regulation predicted exercise for men in a positive direction $(\beta=.26, p<.01)$ but was not related to exercise for women, and introjected regulation was positively but non-significantly associated with 
Table 3 Psychological need satisfaction and self-determined motivation (All five BREQ-2 factors) predicting exercise across gender and age groups (standardized estimates)

\begin{tabular}{|c|c|c|c|c|c|}
\hline & Men & Women & Younger (18-45) & Older (46-78) & Full sample \\
\hline & $n=286$ & $n=805$ & $n=539$ & $n=501$ & $N=1091$ \\
\hline Need $\rightarrow$ Amot. & $-.44(.07)^{* *}$ & $-.44(.04)^{* *}$ & $-.46(.05)^{* *}$ & $-.43(.05)^{* *}$ & $-.44(.06)^{*}$ \\
\hline Need $\rightarrow$ Ext. reg. & $-.14(.07)^{*}$ & $-.30(.04)^{* *}$ & $-.24(.05)^{* *}$ & $-.29(.05)^{* *}$ & $-.26(.04)^{*}$ \\
\hline Need $\rightarrow$ Introj. reg. & $.41(.07)^{* *}$ & $-.03(.05)$ & $.11(.06)^{*}$ & $.08(.06)$ & $.09(.06)$ \\
\hline Need $\rightarrow$ Ident. reg. & $.88(.04)^{* *}$ & $.75(.03)^{* *}$ & $.78(.03)^{* *}$ & $.81(.03)^{* *}$ & $.79(.06)^{*}$ \\
\hline Need $\rightarrow$ Intr. mot. & $.84(.03)^{* *}$ & $.79(.02)^{* *}$ & $.76(.03)^{* *}$ & $.86(.02)^{* *}$ & $.81(.06)^{*}$ \\
\hline Amot. $\rightarrow$ Exercise & $-.10(.09)$ & $.00(.05)$ & $-.02(.06)$ & $-.02(.07)$ & $-.02(.04)$ \\
\hline Ext. reg. $\rightarrow$ Exercise & $.26(.08)^{* *}$ & $-.00(.04)$ & $.04(.05)$ & $.12(.06)^{*}$ & $.07(.05)$ \\
\hline Introj. reg. $\rightarrow$ Exercise & $.12(.10)$ & $-.14(.05)^{*}$ & $-.15(.06)^{* *}$ & $-.06(.06)$ & $-.08(.05)$ \\
\hline Ident. reg. $\rightarrow$ Exercise & $-.06(.16)$ & $.40(.07)^{* *}$ & $.52(.08)^{* *}$ & $.11(.11)$ & $.30(.11)^{*}$ \\
\hline Intr. mot. $\rightarrow$ Exercise & $.16(.14)$ & $.04(.07)$ & $-.06(.08)$ & $.24(.10)^{*}$ & $.08(.06)$ \\
\hline
\end{tabular}

Need = total need satisfaction factor; Amot. = Amotivation; Ext. reg. = External regulation; Introj. reg. = Introjected regulation; Ident. reg. $=$ Identified regulation; Intr. mot. = Intrinsic motivation. ${ }^{*} p<.05 ;{ }^{* *} p<.01$. A complete table with standard errors for the estimates can be obtained by request from the first author

exercise for men but negatively and significantly associated with exercise for women $(\beta=-.14, p<.05)$. Finally, identified regulation predicted exercise for women $(\beta=.40, p<.01)$ but not for men.

When differences in paths between age groups were examined, significant differences were noted in the two paths: identified regulation to exercise $\left(\Delta X^{2}=7.19 / 1 \mathrm{df}\right)$, where the path was stronger and significant for the younger year group $(\beta=-.52 p<>.01)$ compared to the older one $(\beta=.11, p>.05)$, and intrinsic motivation to exercise $\left(\Delta \chi^{2}=5.87 / 2 \mathrm{df}\right)$, where the path was negative and non-significant $(\beta=-.06, p>.05)$ for the young group but positive and significant $(\beta=.24, p<.05)$ for the older one.

Table 4 Correlations between different exercise intensities and latent variables of psychological needs and motivational regulations Exercise intensity

\begin{tabular}{lcccc} 
& Light & Moderate & Strenuous & METS \\
\hline BPNES & & & & \\
Autonomy & -.02 & $.17^{*}$ & $.33^{* *}$ & $.31^{* *}$ \\
Competence & -.07 & $.19^{*}$ & $.46^{* *}$ & $.36^{* *}$ \\
Relatedness & -.07 & .04 & $.31^{* *}$ & $.17^{*}$ \\
Global Need & -.06 & $.18^{*}$ & $.43^{* *}$ & $.35^{* *}$ \\
BREQ-2 & & & & \\
Amotivation & .05 & $-.11^{*}$ & $-.17^{*}$ & $-.16^{*}$ \\
Extrinsic & .05 & .01 & $-.11^{*}$ & -.03 \\
Introjected & -.02 & -.02 & $.12^{*}$ & .03 \\
Identified & -.05 & $.11^{*}$ & $.47^{* *}$ & $.33^{* *}$ \\
Intrinsic & -.04 & $.14^{*}$ & $.36^{* *}$ & $.29^{* *}$ \\
\hline
\end{tabular}

METS = metabolic equivalent of exercise, a total weighted exercise score; global need $=$ total need satisfaction factor; ${ }^{*} \mathrm{p}<.05 ;{ }^{* *} \mathrm{p}<.01$
The mediating effect of autonomous motivation in the association of psychological need satisfaction with exercise The mediating (indirect) effects of controlled and autonomous motivation and the separate BREQ-2 factors are presented in Table 5. In the full sample there was a significant indirect effect of the autonomous motivation $(\alpha \beta=$ 5.42 , $95 \% C I=4.21-6.52$ ), indicating that autonomous motivation acted as a mediating variable in the relationship between psychological need satisfaction and exercise. There was however no indirect effect of controlled motivation. Looking more specifically at the mediating effects of the different BREQ-2 factors, indirect effects were found for amotivation $(\alpha \beta=0.49,95 \% C I=0.06-0.92)$, identified regulation $(\alpha \beta=2.70,95 \% C I=1.05-4.10)$ and intrinsic motivation $(\alpha \beta=1.83,95 \% C I=0.49-3.12)$.

As a consequence of the differences in paths between need, motivation and exercise for men and women, the indirect effects of motivation also differed, in particular for controlled motivation, external regulation and introjected regulation (see Table 5). For example, the indirect effect of controlled motivation was negative and statistically significant for men but positive and non-significant for women. Also, the indirect effect of external regulation was negative and significant for men but positive and non-significant for women and the indirect effect of introjected regulation was positive and statistically significant for men but negative and non-significant for women. However, gender or age did not significantly moderate any of the indirect effects as no Wald Chi-Square tests were significant.

\section{Discussion}

The purpose of the present study was to examine key pathways in a self-determination based model of motivation in exercise, linking satisfaction of psychological 
Table 5 The mediating (indirect) effects of self-determined motivation in the relationship between psychological need satisfaction and exercise across gender and age group

\begin{tabular}{llllll}
\hline $\begin{array}{l}\text { Bootstrap results for indirect effects } \\
\text { Indirect effects }\end{array}$ & Men $a \beta(95 \% C)$ & Women $a \beta(95 \% C l)$ & Younger (18-45) $a \beta(95 \% C l)$ & Older (46-78) $a \beta(95 \% C$ C) & Full sample $a \beta(95 \% C$ C) \\
\hline Controlled motivation & $-1.91^{*}(-6.83-0.37)$ & $0.12(-0.44-0.58)$ & $-0.16(-1.09-0.37)$ & $-0.59(-3.73-0.22)$ & $0.29(-0.44-1.03)$ \\
Autonomous motivation & $4.30^{*}(1.60-6.49)$ & $5.60^{*}(4.31-6.90)$ & $7.28^{*}(4.25-10.14)^{*}$ & $3.98^{*}(2.30-5.66)$ & $2.35^{*}(0.99-3.71)$ \\
Amotivation & $0.71^{*}(0.10-2.27)$ & $0.45(-0.09-0.99)$ & $0.71(-0.29-1.81)$ & $0.43(-0.40-1.45)$ & $0.49^{*}(0.06-0.92)$ \\
External regulation & $-2.27^{*}(-7.60-0.50)$ & $0.16(-0.28-0.57)$ & $-0.13(-1.07-0.44)$ & $-0.49(-2.89-0.26)$ & $-0.39(-1.40-0.11)$ \\
Introjected regulation & $1.48^{*}(0.35-4.26)$ & $-0.10(-0.53-0.26)$ & $-0.10(-1.00-0.20)$ & $-0.05(-0.91-0.18)$ & $-0.09(-0.38-0.02)$ \\
Identified regulation & $2.95(-5.94-7.69)$ & $2.72^{*}(1.57-3.93)$ & $3.06(-0.24-7.56)$ & $2.57(-0.41-4.79)$ & $2.70^{*}(1.05-4.10)$ \\
Intrinsic motivation & $3.17^{*}(0.88-6.05)$ & $1.39(-0.11-2.83)$ & $-0.82(-4.94-3.07)$ & $1.12(-0.38-2.75)$ & $1.83^{*}(0.49-3.12)$ \\
\hline
\end{tabular}

Note: $\alpha \beta=$ product-of-coefficient estimate ( $95 \%$ bias-corrected and accelerated bootstrap $\mathrm{Cl}$ based on 1000 bootstrap resamples); Controlled motivation = external regulation + introjected regulation); Autonomous motivation $=$ identified regulation + intrinsic motivation; * $p<.05$

needs with autonomous motivation and exercise, and specifically to look at how gender and age may moderate these pathways. Moving from first-generation research questions targeting whether relationships exist to secondgeneration research questions focusing on the conditions under which, and when, relationships exist and, finally, to third-generation questions targeting mechanisms of change (mediators) in relationships entails vital steps in the progress of knowledge development in any field [46]. SDTrelated exercise research has typically evolved around firstbut not second- or third-generation research questions, resulting in a gap in the knowledge base regarding what factors moderate and mediate key relationships in the theory. Our main analyses in the full sample revealed that higher need satisfaction predicted autonomous motivation, and that autonomous motivation in turn predicted behavioural outcomes in terms of more exercise. Thus, our results are well in line with general SDT stipulations and previous research (e.g., [13-15, 23, 24, 47]). Moreover, our study advanced previous work by demonstrating differences in paths between need satisfaction, motivation and exercise. For example autonomous motivation, and in particular identified regulation, was a stronger predictor of exercise for women compared with for men, whereas controlled motivation was positively associated with exercise in men but not in women. Overall these findings contribute with interesting information regarding how the theoretically hypothesized associations between need satisfaction, motivation and exercise may be moderated.

In essence, we found that autonomous motivation, but not controlled motivation, mediated the relationship between psychological need satisfaction and exercise. Thus, this study further confirms previous suggestions that the relationship between psychological need satisfaction and outcomes is mediated by motivation [22, 23, 47], adding to the understanding of the underlying mechanisms of SDT constructs and how they can influence behaviour. Because comparable previous studies have demonstrated only partial [25] or no mediating effects of self-determined motivation [26] using non-recommended mediation analysis $[28,29]$, our study seems to be one of the first to demonstrate the mediating effect of self-determined motivation in the relationship between need satisfaction and outcomes in the context of exercise, while also considering moderating effects.

Although the differences across gender and age in the mediation effects of motivation were not statistically significant, there were indications that the potential mechanisms through which need satisfaction influence exercise may differ in direction and strength across subgroups such as gender. In order to design effective exercise interventions, previous work has proposed that "one size may not fit all" [48] and that further investigation is needed concerning cross-study differences in SDTrelated relationships regarding gender [33] as well as age and other potential moderating factors [14]. Most paths in the model were invariant according to SDT [11], but at some points we found pathway differences between age and gender groups, not only using controlled and autonomous motivation factors but also for the subscale regulations (identified and intrinsic regulations, as well as amotivation), which paints a slightly more complex picture of the mechanisms responsible for exercise behaviour. As stated by Hayes [49], "...an analysis that ignores the potential contingencies and boundary conditions of an effect is going to result in a greater oversimplification of complex processes relative to an analysis that acknowledges that complexity by formally modelling it..." (p. 327). In this way, our study represents a primary attempt to create a better understanding of the SDT process model in terms of behavioural outcome. Since the nuts and bolts of how this would inform intervention design more specifically still remain unclear, the results of this study highlight the need for further inquiry regarding possible moderating effects. We therefore agree with previous research that addressing age and gender issues could benefit practice [14] and propose future studies to more thoroughly examine notions of both mediating and moderating effects. 
Offering simple explanations for the demonstrated moderating effects of age and gender is not easy, and due to the exploratory nature of the moderation analyses such clarifications would seem quite premature. Previous research mainly concerns mean-level observations, and does not contribute a rich frame of reference for explaining the specific differences in paths between men and women and different age groups (e.g., [14, 33]). Nevertheless, the choice to examine these paths is based on some preliminary thoughts that should be expanded on. For example, there is reason to believe that factors like age and gender could influence the means by which, for example, basic needs are satisfied [11, 17], and it is likely that people's reasons for exercise change over their lifespans [30]. Although both identified regulation and intrinsic motivation are highly self-determined and integrated into the self, previous research has shown that older adults have more intrinsically oriented exercise goals and motives than younger adults do (e.g., $[31,50])$. Accordingly, the current results (demonstrating that intrinsic regulation positively predicts exercise only for older adults, whereas identified regulation was a stronger exercise predictor among younger adults) might support these previous mean-level studies by indicating that the older adults in this sample were somewhat more autonomously regulated than the younger adults. Furthermore, these mediating effects might not be moderated by age per se, but perhaps age serves as a proxy in terms of different stages in life possibly offering different opportunities and barriers to choose between leisure time activities with various degrees of need support (or need thwarting). Such arguments imply that the moderated mediation effects found in this study merely represent a first step towards the exploration of possible underlying mechanisms and other potential mediators. Further investigation of moderated mediation effects is needed in order to offer deeper knowledge of how to address these mechanisms, perhaps by studying how the social contexts might differ for the different sub groups (men/women and younger/older adults) and thereby may or may not support need fulfilment related to barriers and opportunities in the social environment. It should be noted that when the sample was split into three age groups (younger, middle-aged and older adults) the findings on age differences remained essentially similar to those based on two age groups.

In regard to gender, the present results add to the inconsistency shown in previous studies on mean level by displaying more controlled (external and introjected) regulations predicting exercise for men, whereas more autonomous (identified) regulation predicted exercise for women, which is in line with the study by $\mathrm{Li}$ [32] but quite the opposite of the conclusions in the review by Teixeira, Carraca [14], and non-consistent with, for example, research by Guérin, Fortier [33]. These inconsistencies point to a clear need to further examine gender-related differences in how regulations are associated with exercise; and the arguments above regarding other potential underlying dimensions of age moderating the mediation effects also apply to the discussion of gender (e.g., social-environmental factors).

There is also reason to mention something about relations between the different exercise intensities, psychological needs and motivational regulations. Light exercise was not found to be related with needs or motivational regulations and moderate exercise was only weakly related to two needs and three regulations. Strenuous exercise, however, was shown to be moderately associated with all three needs, identified regulation and intrinsic motivation and also significantly but weakly associated with the other three motivation regulations. These results are in line with previous findings on exercise intensity and motivational regulations (e.g., [51, 52]). As has been forwarded by others [50], light and moderate exercise may incorporate activities such as walking and cycling that are more habitual in nature and therefore will be less affected by cognitive processing compared to more structured and strenuous types of exercise. Also, strenuous activities may require more engagement, planning and self-regulation skills than light and moderate activities. Future studies should further explore different predictors/models for the different intensities.

The strong competence-autonomy association in our study most probably resulted in the suppressor effect, which was demonstrated by an unexpected and, from a theoretical viewpoint illogical, negative association in the model between autonomy and autonomous motivation. The suppressor effect was handled in our study by collapsing the three needs into one global latent need satisfaction factor. This modified model fit data well, which is in line with the findings in previous work (e.g., $[20,21]$ ) that a single global need satisfaction factor could explain latent variables representing autonomy, competence and relatedness. Moreover, these results are also in line with the assertion $[17,53]$ that the satisfaction of the three needs is complementary; that is, that the satisfaction of one need (e.g., autonomy) can occur only if the other needs are also satisfied.

\section{Limitations and future directions}

The current results should be interpreted cautiously with regard to methodological differences in previous studies (i.e., traditional versus more advanced methods like SEM), and the somewhat contradictive results call for further inquiries concerning these moderators, using advanced analysis and various samples. The assumption of linear relations between motivational and behavioural variables should also be taken into consideration, since associations could be non-linear. Interpretation should also consider the specific sample. First, the sample consists of members 
of a web-based exercise programme focused on step contests and weight loss, which might have impacted participative motives, regulations and preferences; second, the relatively high mean age (45 years) combined with the female dominance could also have affected the results (e.g., women being more autonomous due to higher age); and third, this specific population of older adults might not be representative of this age group in general (e.g., these webbased exercise services might attract older adults with certain capacities and/or characteristics). Furthermore, women seem be more inclined to join web-based PA interventions (see e.g., [54-56]), which might serve as a complementary reason why women were more autonomously motivated in this study than men were. It is also possible that men are mainly attracted by the competitive features of the web service, which might be expected to pull for more controlled motivations per se. The primary limitation of the study, however, is the cross-sectional design. Therefore, even though proper analyses were used [8] also for crosssectional data [57], and although a direction is implied in our analytical model (e.g., need satisfaction predicting motivation), we cannot rule out the possibility of reversed causation. Moreover, drawing conclusions about mediation analyses based on cross-sectional data may be misleading because mediation consists of processes that unfold over time [58]; future studies should therefore further examine whether the mediating role of autonomous motivation will hold longitudinally. Also, using the revised four-point Likert BREQ-2 scale instead of the original five-point scale may have resulted in less variation and higher total scores, primarily for amotivation and external regulation. Nevertheless, the analyses showed the factor structure to be stable also with the revised four-point scale.

Finally, even though a comparatively reliable [59] and valid [60] self-report measure of exercise was used, it is unquestionably less reliable than objective measures. On the other hand, we employed a large e-health based sample of non-clinical middle-aged adults, who are more rarely studied in the context of motivation and exercise. In addition, we conducted age- and gender-specific analyses, contributing new information in terms of when and for whom the different paths in the SDT model exist or are stronger/ weaker, but more work is needed in order to understand how such mediating and moderating effects and mechanisms adds to the growing evidence of SDT utility [12-15] and potential practical implications. Making the best use of SDT as a kind of compass in constructing exercise interventions could assist a smoother and more sustainable transition from inactivity to activity for inactive individuals and our results highlight the importance for researchers in the future to examine the effect of potentially relevant moderating factors that may influence the different paths in the SDT-based models, rather than controlling these variables (e.g., age and gender), as typically tends to be done.

\section{Conclusions}

Based on sophisticated mediation analysis, the results support the hypothesized associations between latent constructs and exercise behaviour in the related steps of the SDT process model. Autonomous motivation was found to act as a mediating variable in the relationship between psychological need satisfaction and exercise. We also found some of the paths to differ across age and gender, which could indicate that mechanisms in the SDT process model could vary (qualitatively) depending on subgroup, which calls for further exploration in future research.

\section{Competing interests}

The authors declare that they have no competing interests.

\section{Author's contributions}

KWJ participated in the study design, data collection, drafted and finalized the manuscript. ML supervised the writing process, contributed to the manuscript writing and carried out the statistical analyses. Al participated in data collection and the statistical analyses. All authors read and approved the final manuscript.

\section{Acknowledgements}

This research has been financially supported by the Centre for Person-Centred Care at Gothenburg University; Halmstad University; Health Technology Centre Halland; Tappa Service AB and the European Regional Development Fund.

\section{Author details}

${ }^{1}$ Research on Welfare, Health and Sport, Halmstad University, PO Box 823, SE 30118 Halmstad, Sweden. ${ }^{2}$ Department of Psychology, University of Gothenburg, PO Box 500, SE 40530 Gothenburg, Sweden. Department of Food and Nutrition, and Sport Science, University of Gothenburg, Box 100, SE 40530 Gothenburg, Sweden. ${ }^{4}$ Centre for Person Centered Care, University of Gothenburg, Box 457, SE 40530 Göteborg, Sweden.

Received: 30 June 2014 Accepted: 11 May 2015

Published online: 20 May 2015

\section{References}

1. WHO. The European health report. 2009, Health and health systems W.H.O.R.O.f. Europe. Copenhagen: World Health Organization, Regional Office for for Europe; 2009.

2. WHO. The European Health Report 2012, W.H.O.R.O.f. Europe. Copenhagen: World Health Organization, Regional Office for for Europe; 2013. Physical Activity.

3. Guidelines Advisory Committee. Physical activity guidelines advisory committee report. Washington DC, U.S.: Department of Health and Human Services; 2008

4. Ezzati M, Lopez AD, Rodgers A, Murray CJL. Mortality and burden of disease attributable to individual risk factors. In: Bull FC, Armstrong TP, Dixon T, Ham S, Nieman A, Pratt M, editors. Comparative Quantification of Health Risks Global and Regional Burden of Disease Attributable to Selected Major Risk Factors. Geneva: World Health Organization; 2004.

5. WHO Insufficient physical activity 2008. Prevalence of insufficent physical activity, ages 15+, both ages. 2011. Online resource, 2014-0318 http://gamapserver.who.int/gho/interactive_charts/ncd/risk_factors/ physical_inactivity/atlas.html.

6. Baranowski T, Jago R. Understanding the mechanisms of change in children's physical activity programs. Exerc Sport Sci Rev. 2005;33(4):163-8.

7. Rhodes RE, Pfaeffli LA. Mediators of physical activity behaviour change among adult non-clinical populations: a review update. Int J Behav Nutr Phys Act. 2010;7:37.

8. Cerin E, Mackinnon DP. A commentary on current practice in mediating variable analyses in behavioural nutrition and physical activity. Public Health Nutr. 2009:12(8):1182-8.

9. Lubans DR, Foster C, Biddle SJ. A review of mediators of behavior in interventions to promote physical activity among children and adolescents. Prev Med. 2008;47(5):463-70.

10. Deci EL, Ryan RM. Intrinsic motivation and self-determination in human behavior. New York: Plenum Press; 1985. 
11. Deci EL, Ryan RM. The "what" and "why" of goal pursuits: Human needs and the self-determination of behavior. Psychol Inq. 2000;4:227-68.

12. Ntoumanis N, Thogersen-Ntoumani C, Deci EL, Ryan RM, Duda JL, Williams GC. Self-determination theory applied to health contexts: a meta-analysis. Perspect Psychol Sci. 2012;7(4):325-40

13. Hagger MS, Chatzisarantis NLD. Self-determination theory and the psychology of exercise. Int Rev Sport Exerc Psychol. 2008;1(1):79-103.

14. Teixeira PJ, Carraca EV, Markland D, Silva MN, Ryan RM. Exercise, physical activity, and self-determination theory: a systematic review. Int J Behav Nutr Phys Act. 2012;9:78.

15. Fortier MS, Sweet SN, Tulloch H, Blanchard CM, Sigal RJ, Kenny GP, et al. Promoting physical activity: development and testing of self-determination theory-based interventions. Int J Behav Nutr Phys Act. 2012;9:20.

16. Fortier MS, Wiseman E, Sweet Shane N, O'Sullivan T, Blanchard CM, Sigal RJ, et al. A moderated mediation of motivation on physical activity in the context of the physical activity counseling randomized control trial. Psychol Sport Exerc. 2011;12(2):71-8.

17. Ryan RM, Deci EL. Overview of self-determination theory: An organismic dialectical perspective. In: Ryan RM, Deci EL, editors. Handbook of selfdetermination research. Rochester: University of Rochester Press; 2002.

18. Vallerand RJ. A hierarchical model of intrinsic and extrinsic motivation for sport and physical activity. In: Chatzisarantis MSHNLD, editor. Intrinsic Motivation and Self-Determination in Exercise and Sport. Champaign, IL: Human Kinetics; 2007.

19. Vallerand RJ. Toward a hierarchical model of intrinsic and extrinsic motivation. In: Zanna MP, editor. Advances in experimental social psychology. New York: Academic; 1997.

20. David Markland VJT. Need support and behavioural regulations for exercise among exercise referral scheme clients: The mediating role of psychological need satisfaction. Psychol Sport Exerc. 2010;11:91-9.

21. Hagger MS, Chatzisarantis NLD, Harris J. From psychological need satisfaction to intentional behavior: testing a motivational sequence in two behavioral contexts. Personal Soc Psychol Bull. 2006;32:131-8.

22. Vallerand RJ, Losier GF. An integrative analysis of intrinsic and extrinsic motivation. J Appl Sport Psychol. 1999;11:142-69.

23. Williams GC, McGregor HA, Zeldman A, Freedman ZR, Deci EL. Testing a self-determination theory process model for promoting glycemic contro through diabetes self-management. Health Psychol. 2004;23(1):58-66.

24. Silva MN, Markland D, Carraca EV, Vieira PN, Coutinho SR, Minderico CS, et al. Exercise autonomous motivation predicts 3-years weight loss in women. Med Sci Sports Exerc. 2011;43(4):728-37.

25. Edmunds J, Ntoumanis N, Duda JL. A test of self-determination theory in the exercise domain. J Appl Soc Psychol. 2006;36(9):2240-65.

26. McDonough MH, Crocker PRE. Testing self-determination as a mediator of the relationship between psychological needs and affective and behavioral outcomes. J Sport Exerc Psychol. 2007;29:645-63.

27. Baron RM, Kenny DA. The moderator-mediator variable distinction in social psychological research: conceptual, strategic, and statistical considerations. J Pers Soc Psychol. 1986;51:1173-82.

28. Cerin E. Ways of unraveling how and why physical activity influences mental health through statistical mediation analyses. Ment Health Phys Act. 2010;3:51-60.

29. Preacher KJ, Hayes AF. Asymptotic and resampling strategies for assessing and comparing indirect effects in multiple mediator models. Behav Res Methods Achiev Exp Pathol. 2008;40:879-91.

30. Miller AM, Iris M. Health promotion attitudes and strategies in older adults. Health Educ Behav. 2002;29:249-67.

31. Brunet J, Sabiston CM. Exploring motivation for physical activity across the adult lifespan. Psychol Sport Exerc. 2011;12(2):99-105.

32. Li F. The exercise motivation scale: its multifaceted structure and construct validity. J Appl Sport Psychol. 1999;11(1):97-115.

33. Guérin E, Fortier MS, Sweet S. A meta-analysis of the influence of gender on self-determination Theory's motivational regulations for physical activity. Can Psychol. 2012;53(4):291-300.

34. Vlachopoulos SP, Michailidou S. Development and initial validation of a measure of autonomy, competence, and relatedness in exercise: the basic psychological needs in exercise scale. Meas Phys Educ Exerc Sci. 2006:10(3):179-201

35. Vlachopoulos SP. The basic psychological needs in exercise scale: measurement invariance over gender. Struct Equ Model. 2008;15:114-35.
36. Markland D, Tobin VJ. A modification of the behavioral regulation in exercise questionnaire to include an assesment of amotivation. J Sport Exerc. 2004;26:191-6

37. Sebire SJ, Standage M, Vansteenkiste M. Development and validation of the goal content for exercise questionnaire. J Sport Exerc Psychol. 2008;30(4):353-77

38. Godin G, Shephard RJ. A simple method to assess exercise behavior in the community. Can J Appl Sport Sci. 1985;10(3):141-6.

39. Brislin RW. The wording and translation of research instruments. In: Berry WJLJW, editor. Field methods in cross-cultural research. Sage: Thousand Oaks; 1986. p. 137-64.

40. Ericsson K, Simon H. Protocol analysis: verbal reports as data. 2 ed. Boston: MIT Press; 1993

41. Preacher KJ, Hayes AF. SPSS ans SAS procedures for estimating indirect effects in simple mediation models. Behav Res Methods. 2004;36(4):717-31.

42. Hu L-T, Bentler PM. Cutoff criteria for fit indexes in covariance structure analysis: conventional criteria versus new alternatives. Struct Equ Model. 1999;6(1):1-55.

43. Bentler PM. Comparative fit indexes in structural models. Psychol Bull. 1990;107:238-46

44. Browne MW, Cudeck R. Alternative ways of assessing model fit. In: Long KABJS, editor. Testing structural equation models. Newbury Park: Sage; 1993. p. 136-62

45. Cheung GW, Rensvold RB. Evaluating goodness-of-fit indexes for testing measurement invariance. Struct Equ Model. 2002:9:233-55.

46. Zanna MP, Fazio RH. The attitude-behaviour relation: Moving toward a third generation of research. In: Zanna MP, Higgins ET, Herman CP, editors. Social Psychology. Hillsdale NJ: Erlbaum; 1982.

47. Ryan RM, Deci EL. Active human nature: Self-Determination theory and the promotion and maintenance of sport, exercise, and health. In: Chatzisarantis MSHNLD, editor. Intrinsic Motivation and Self-Determination in Exercise and Sport. Champaign, IL: Human Kinetics; 2007.

48. Gallagher P, Yancy Jr WS, Swartout K, Denissen JJ, Kuhnel A, Voils Cl. Age and sex differences in prospective effects of health goals and motivations on daily leisure-time physical activity. Prev Med. 2012;55(4):322-4.

49. Hayes AF. Introduction to mediation, moderation, and conditional process analysis: a regression-based approach. New York: Guilford; 2013.

50. Beck F, Gillison F, Standage M. A theoretical investigation of the development of physical activity habits in retirement. Br J Health Psychol. 2010;15:663-79.

51. Edmunds J, Ntoumanis N, Duda JL. Examining exercise dependence symptomatology from a self-determination perspective. J Health Psychol. 2006;11(6):887-903.

52. Silva MN, Vieira PN, Coutinho SR, Minderico CS, Matos MG, Sardinha LB, et al. Helping overweight women become more active: need support and motivational regulations for different forms of physical activity. Psychol Sport Exerc. 2010;11:591-601.

53. Wilson PM, Rodgers WM. Self-determination theory, exercise and well-being. In: Chatzisarantis MSHNLD, editor. Intrinsic motivation and selfdetermination in exercise and sport. Champaign, IL: Human Kinetics; 2007.

54. Brouwer W, Oenemai A, Raat H, Crutzen R, de Nooijer J, de Vries N, et al. Characteristics of visitors and revisitors to an internet delivered computertailored lifestyle intervention implemented for use by the general public. Health Educ Res. 2010;25(4):585-95.

55. Dawson KA, Tracey J, Berry T. Evaluation of work place group and internet based physical activity interventions on psychological variables associated with exercise behaviour change. J Sports Sci Med. 2008;7:537-43.

56. Napolitano MA, Fotheringham M, Tate D, Sciamanna C, Leslie E, Owen N, et al. Evaluation of an internet-based physical activity intention: a preliminary intervention. Ann Behav Med. 2003:25:92-9.

57. Kline RB. Principles and practice of structural equation modeling. New York, NY: Guilford Press; 1998.

58. Maxwell SE, Cole DA. Bias in cross-sectional analyses of longitudinal mediation. Psychol Methods. 2007;12:23-44

59. Jacobs DTR, Ainsworth BE, Hartman TJ, Leon AS. A simultaneous evaluation of 10 commonly used physical activity questionnaires. Med Sci Sports Exerc. 1993:25:81-91.

60. Wilson PM, Mack DE, Gunnell KE, Gregson P, Cheung S, Rimmer S. Assessing leisure-time physical activity in young adults with the Godin leisure-time exercise questionnaire: normative values, gender differences, and nonresponse error. Ann Behav Med. 2010;39:188. 